\title{
Using Ultrasonic Haptics Within an Immersive Spider Exposure Environment to Provide a Multi-Sensorial Experience
}

\author{
Daniel Brice*, Zara Gibson, Fintan McGuinness and Karen Rafferty \\ School of Electronics, Electrical Engineering and Computer Science, Queen's University Belfast, Belfast, United Kingdom
}

A proof of concept virtual reality system is presented that integrates ultrasonic feedback sensations to provide a demonstrative virtual reality exposure therapy environment containing multiple scenarios with virtual spiders. This system and environment were utilised to conduct a study containing 35 participants with the goal of investigating the effect the environment could have on their level of anxiety. This level of anxiety was measured in three different forms: changes in frontal asymmetry analysis of EEG data, changes in skin conductance levels and subjective units of distress. The Fear of Spiders Questionnaire was used to determine which participants in the study reported to be

OPEN ACCESS

Edited by:

Daniele Leonardis,

Sant'Anna School of Advanced

Studies, Italy

Reviewed by:

Gabriele Fusco,

Sapienza University of Rome, Italy

William Frier,

Ultraleap Ltd., United Kingdom

${ }^{*}$ Correspondence:

Daniel Brice

dbrice01@qub.ac.uk

Specialty section:

This article was submitted to

Haptics,

a section of the journal

Frontiers in Virtual Reality

Received: 10 May 2021

Accepted: 27 July 2021

Published: 10 August 2021

Citation:

Brice D, Gibson Z, McGuinness F and Rafferty K (2021) Using Ultrasonic Haptics Within an Immersive Spider Exposure Environment to Provide a

Multi-Sensorial Experience.

Front. Virtual Real. 2:707731.

doi: 10.3389/frvir.2021.707731 moderately afraid of spiders. For these participants all three measurement forms for anxiety showed statistically significant increases in a comparison between baseline and scenarios with the virtual spiders. A statistically significant correlation between scores on the Fear of Spiders Questionnaire and changes in anxiety shows the system to have had a greater effect on the anxiety levels of those who were more afraid of spiders, than those who were not. There was also a statistically significant correlation discovered between immersion and increase in anxiety, highlighting the significance of immersion in future virtual reality exposure therapy applications.

Keywords: virtual reality, haptics, immersion, VRET, ultrasonic feedback, spiders

\section{INTRODUCTION}

A common and effective method for treating phobias is gradual exposure therapy, which entails a patient being subjected to multiple scenarios with increasing levels of anxiety associated Ollendick and Davis III (2004). Over recent decades advances in virtual reality (VR) technology have enabled the digitisation of exposure therapy, termed virtual reality exposure therapy (VRET), which has been shown to be a preferred choice for people over the traditional method Garcia-Palacios et al. (2007); Rothbaum et al. (1995). VRET has also been praised by therapists, who have shown willingness to utilise it Segal et al. (2011). VRET relies on the latest immersive technologies to produce a strong sense of presence for users. However, the haptic technologies involved in VRET for the fear of spiders have remained more traditional, using toy props.

A study with a sample of 35 participants consisting of university staff and students was carried out to demonstrate the capability of VR to enhance the sense of spider related anxiety during exposure, a requirement of VRET. The use of ultrasonic feedback for contact with spiders in VR was introduced for a condition in the study to provide quantitative user feedback on its realism and accuracy. The 
study has a greater number of participants than most similar studies and expands upon the existing literature by utilising a greater number of anxiety measures, offering strong evidence of the capability of VR to enhance the sense of anxiety during exposure to virtual spiders.

The main contribution of the paper is a study with 35 participants utilising both objective and subjective indicators that demonstrates how effectively VR can be used to enhance the sense of spider-related anxiety within those who are somewhat afraid of spiders, as well as the relationship presence has with this anxiety. Secondary contributions of the paper are the design and implementation of an enhanced VR experience through integration of ultrasonic technology with stereoscopic $3 \mathrm{D}$ visual $\mathrm{VR}$ to provide an immersive multisensory system, enabling studies into the effects of VR spiders with tactile sensations to be conducted. As well as quantitative feedback, regarding accuracy and realism, on users' first experiences with ultrasonic feedback for spider related stimuli in VR.

\section{RELATED WORK}

\subsection{Inducing Anxiety With Immersive Virtual Reality}

VR is known to have the ability to affect emotions such as state anxiety Riva et al. (2007). This is a necessary element of VRET, where it is desirable for virtual environments to induce anxiety similarly to in vivo situations.

Research carried out by Stinson and Bowman (2014) demonstrates the efficacy of VR to induce anxiety during high pressure virtual sports scenarios. In their study 28 participants took part in a goalkeeping task in VR with known anxiety triggers. Their results demonstrated significant differences in both subjective and objective measures of anxiety, obtained by measuring skin conductance, from the baseline condition. Their work further validates the utilisation of skin conductance as a means of assessing anxiety. The research presented in this paper utilises these same validated techniques for measuring anxiety in VR, but it is applied to anxiety concerning spiders instead of high pressure sporting scenarios.

Yeh et al. (2018) conducted a study where participants experienced claustrophobic environments in both VR and AR with subjective and objective measure of anxiety. Their findings showed that participants experienced a degree of anxiety in the VR condition. They also noted that participants reported issues with realism in aspects such as not being able to see legs when they looked down in VR. In their work skin conductance is used as one of the objective measures of anxiety alongside subjective reporting. The work presented in this paper uses these same techniques, but applies them to spider related fear, as opposed to claustrophobia.

Juan and Pérez (2010) developed claustrophobic scenarios where large virtual holes were formed in the floor for both AR and VR conditions for a user study investigating presence and anxiety. Subjective measures from their VR condition showed no change in anxiety from participants during the experiment. They report that this is most likely due to not having participants who suffer from claustrophobia. Moderate correlations were reported between reports of presence and reports of anxiety for the VR condition. Our research aims to further investigate this link of anxiety and immersion, but applied to anxiety regarding spiders and not claustrophobia. Their work was unable to demonstrate that anxiety could be raised in non-phobic participants. However, in this paper evidence is provided to show that participants do not need to have a phobia for their anxiety levels to be raised by the relevant stimuli in $\mathrm{VR}$.

A study was conducted by Brouwer et al. (2011) with nine participants where stress was induced in VR by means of visiting virtual cities after bomb explosions and providing negative feedback to participants through VR. In their study subjective measures of stress were obtained alongside objective measures using alpha asymmetry from EEG recordings amongst other methods. The study successfully demonstrated the capability of VR to induce anxiety, as well as contributing evidence of the ability of EEG asymmetry analysis for measures of anxiety in VR. It is noteworthy that the study contained haptics in the form of a vibrotactile vest, though the sole effects of the haptics on anxiety were not investigated. The paper provides validation in the utilisation of asymmetry analysis on the alpha frequency band as a means of measuring anxiety in VR, justifying its inclusion in our study as a measurement technique.

Another study carried out by Kwon et al. (2013) looked at social anxiety induced within simulated VR job interviews. Specifically, investigations were carried out to determine the effect of immersion on the induced anxiety by varying graphical quality and immersive technology used. The findings of the study suggest that immersion has a big impact on the anxiety experienced by users. The authors of the work state that there must be a degree of physical immersion involved to maintain anxiety levels across a course of exposure in VR. The significant effect of immersion on anxiety induced was also investigated by Pallavicini et al. (2013) who conducted a study where participants experienced classroom scenarios through different forms of media, such as VR. To determine the impact of immersion on anxiety they introduced a VR condition with intentional bugs to reduce the level of presence and compared this to a fully functioning VR condition. Their results showed that these breaks in immersion significantly reduced the effectiveness of VR in inducing anxiety within participants. The main takeaway from their study was that without high levels of presence VR becomes unsuitable as a form of media for inducing anxiety. The research presented in asks similar questions as both of these studies by also attempting to increase anxiety levels of participants and correlating subjective reports of participants' level of presence with reports of their anxiety levels related to spiders instead of social anxiety.

A large-scale study investigating the relationship between presence and anxiety in clinical and non-clinical environments within VR was conducted with 210 students by Alsina-Jurnet et al. (2011). One of the main findings of the study was that for anxious participants, a demographic similar to those who would use VRET, there was a strong relationship between presence and anxiety induced in VR. Participants only experienced anxiety 
when they also reported a high level of presence. Our study similarly measures both anxiety and immersion to determine if there is a link between the two.

Various other studies have also determined that levels of immersion and presence have an effect on anxiety Kwon et al. (2013); Robillard et al. (2003); Bouchard et al. (2008); Diemer et al. (2015); Krijn et al. (2004). From the existing literature it is evident that VR has strong potential to induce anxiety using simulated content. Furthermore, these studies suggest there is a relationship between the anxiety and level of presence experienced during virtual exposure. This highlights the significance of immersion in VRET design and indicates that developers of such applications should ensure high levels of immersion are achieved in order to induce the appropriate levels of anxiety effectively. Technological improvements are one of the means by which immersion is enhanced with newer VRET models. However, efforts to improve immersion may also be done by providing additional multisensory feedback such as in this study.

The majority of the presented literature has shown efficacy of inducing anxiety by simulating immersive environments. The research presented in this paper further enriches this literature by utilising advances in state of art immersive technologies. In doing so it provides evidence from a study with a wide variety of anxiety measurements, a high performance VR headset, a large number of participants and a state of the art haptic platform.

\subsection{Haptics in Arachnophobia Virtual Reality Exposure Therapy}

A study on existing VRET systems recommends increasing realism and presence through the addition of multisensory feedback in the form of haptics, the sense of touch Price and Anderson (2007). Traditionally in the domain of arachnophobia VRET these haptics have been provided through the addition of props, usually in the form of static 3D objects or toys, as shown in various studies Garcia-Palacios et al. (2002); Hoffman (1998); Hoffman et al. (1996, 1998); Carlin et al. (1997).

The effect of haptics on anxiety and presence in spider VRET was measured during a study carried out by Hoffman et al. (2003). The study showed that a condition with haptics was more effective at inducing anxiety than a control and similar scenario without haptics. In this study not only were anxiety and presence levels elevated for conditions with haptics, but they also found that participants in the haptics conditions were able to approach real spiders following VRET with more confidence and exhibited less anxiety than those who were in the non-haptic VRET condition. Another study by Carlin et al. (1997) similarly looked at the effectiveness of combining tactile feedback with VR for spider phobia exposure. In their study a furry toy spider was used to provide tactile feedback. The results clearly indicated that combining tactile feedback with VR produces high levels of anxiety. In their paper the authors explain that the lines between reality and virtual reality can be blurred through tactile augmentation, resulting in more effective transfer of training from VRET. Similar work carried out by Peperkorn and Mühlberger (2013) also delivered results in a study where spider related content was provided in VR with visual only, tactile only and combined conditions for participants in a randomised order. The findings of the study were that the greatest levels of anxiety and presence were found when combining tactile feedback with the visual counterparts in VR. Although the use of haptics was clearly shown to be effective in all of these studies, the use of toy props for spiders requires constant technician intervention and scalability is constrained by the methodology employed, e.g. different numbers, sizes and movements of spiders. For this reason we aim to provide ultrasonic feedback for spiders in an attempt to increase immersion, resulting in an increased ability to invoke the necessary levels of anxiety.

Very few studies have provided tactile feedback using technological haptics systems in the design of an arachnophobia VRET. One attempt to implement a haptics system for spiders in VR was carried out by Cavrag et al. (2014) featured a Novint Falcon haptic device to provided virtual force feedback for interactions with spiders on a computer, though the effects of this were not measured in a study. One of the limitations of their system design is that users are required to constantly hold a stylus attached to the Novint Falcon. Presence was not measured during the study. However, this interaction methodology would limit VRET users to interactions which consist of touching virtual objects with the stylus, an interaction which is less natural than direct, stylus-free ones between the user's hand and virtual objects.

Recently work was carried out by Kurscheidt et al. (2019) to develop a vibrotactile arm-sleeve to produce tactile feedback for spiders crawling across the arm. The system provided feedback through a number of vibrating motors at fixed locations across the sleeve controlled by a Raspberry Pi. Currently the efficacy of their system has not yet been tested in any studies. Their efforts in incorporating modern haptic solutions to arachnophobia VRET are commendable. However, the design concept of a wearable interface does raise a few issues where end users may find themselves suffering anxiety attacks due to simulated content and wanting to remove all apparatus quickly and safely. We would claim that it is more natural to provide a haptics system where the user is not permanently attached to additional devices, such as an ultrasonic feedback platform. This is so that in the event of the user feeling too anxious they may quickly disengage from the system.

Over the last decade advances in haptics technology has resulted in the development of ultrasonic, tactile feedback interfaces such as Ultrahaptics boards from Bristol University Carter et al. (2013). These interfaces avail of a phenomenon called acoustic radiation, where ultrasound transducers are modulated, resulting in mid-air focal points of the waves Iwamoto et al. (2008). Studies have shown that these focal points can be perceived with high fidelity as slight shear forces under the epidermis Hoshi et al. (2010); Long et al. (2014). To date there are no known examples of published work utilising ultrasonic feedback for spider sensations.

Although many of the studies in the existing literature have suggested that the role of haptics is significant in immersive VR for spiders, the vast majority of studies have used primitive techniques for haptics, most commonly using toy spiders. 


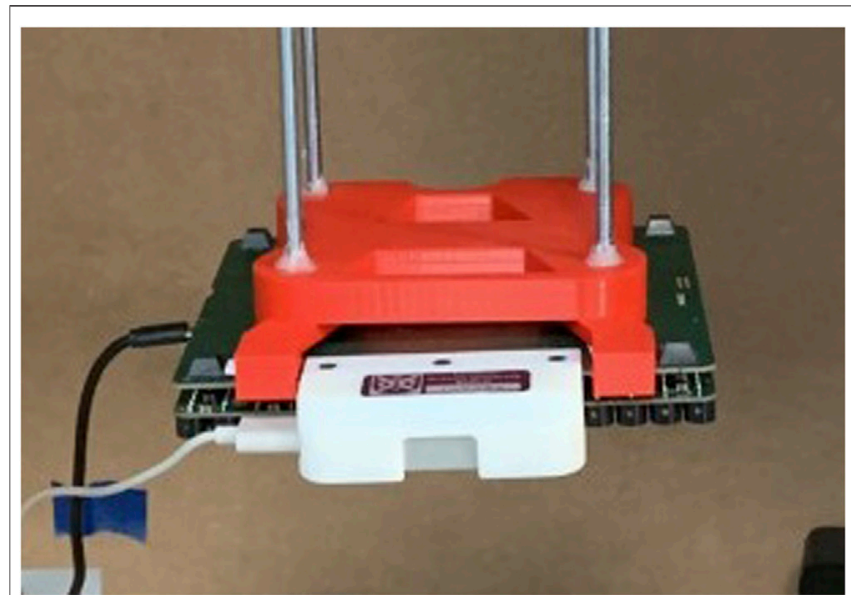

FIGURE 1 | Configuration of haptics module.

Though these techniques have been effective in the past, they are limiting in principle due to being inanimate physical objects which are usually fixed in space. Our study has provided a state of art integration of ultrasonic, tactile feedback for virtual spiders and is the only study known by the authors to do so. This technique offers greater control over the tactile feedback for the spiders by enabling the VR application to change their location, size and movement direction. The haptic platform introduced also offers a greater level of scalability and control than using physical props, enabling feedback to be provided for multiple spiders, flying insects and much more by simply changing the code.

\section{METHODOLOGY}

\subsection{Goals}

The primary goal of the user study was to determine if a VR system with ultrasonic, tactile feedback for virtual spider-related stimuli could have an overall effect on the anxiety levels, measured using skin conductance, SUDS and EEG, of those with atleast a mid-level fear of spiders. A secondary goal was to determine whether the change in anxiety could be attributed to the simulated content and not to outside factors. The third goal of the study was to find if there is a relationship between a participant's level of anxiety and their sense of presence in the virtual environment. A final goal was to gather initial quantitative feedback on the realism and accuracy of the ultrasonic haptic feedback used in the study.

\subsection{Participants}

A total of 35 participants were recruited from a population within the university's science and engineering faculty. Twenty three (66\%) of these participants were male and $12(34 \%)$ of them female. The mean age of the participants was 27 years $(S D=7.66)$ ranging between individuals from 20 to 57. A group of 17 (49\%) participants with at least mid-level fear of spiders was identified by those who scored above 15 in the pre study FSQ, using the same FSQ score cut-off as another study on fear of spiders Wagener and Zettle (2011). None of the participants had a phobia of spiders.

\subsection{Equipment}

Visualisation was provided through a wired HTC VIVE HMD, mounted with side straps on top of the EEG soft cap. An Evaluation Kit from Ultrahaptics was mounted facing downwards on a custom gripper ( $3 \mathrm{D}$ printed), as shown in Figure 1, suspended within a $60 \times 60 \times 60 \mathrm{~cm}$ laser cut MDF box with a black curtain front. Tactile feedback was produced from the ultrasonic transducers in the form of a single circular sensation that is focussed to co-locate with the virtual spider when it was in contact with the participant's hand. The size of the sensation varied according to the size of the spider; this ranging from a diameter of $15-35 \mathrm{~mm}$. Hand tracking was carried out using a Leap Motion hand tracker connected to the Ultrahaptics board in order to provide participants with a visualisation for their hands.

The virtual environment was developed in Unity 3D (v2018.4.6) and contained a chair in front of a desk and box aligned with their real-world counterparts, as shown in Figure 2. Variants of spider size were built into the system with approximate sizes: very small spider, small spider and big spider scaled at 15, 35 and $80 \mathrm{~mm}$, respectively. All the spiders were dark brown in colour without 3D texture such as hairs. Each spider was animated with slow, foward movements and rotations. Spiders on webs had animations to crawl up and down web strings. With these spiders seven scenarios were designed to provide a virtual experience of placing a hand into the box without a spider touching it:

- Spider in bottle (SIB)-A small spider in a closed bottle.

- Small spider far (SSF)-A small spider crawling on the far side of the box.

- Small spider near (SSN)-A small spider crawling near the participant's hand.

- Big spider far (BSF)-A big spider crawling on the far side of the box.

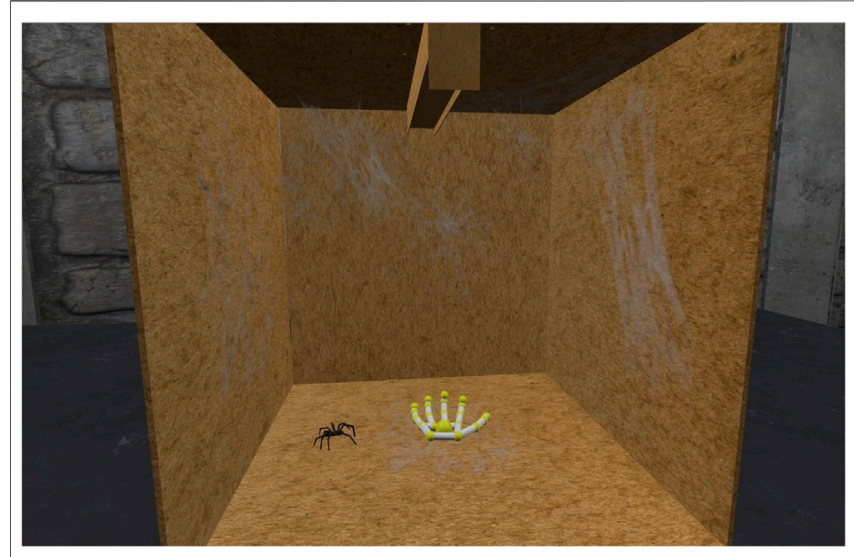

FIGURE 2 | Virtual environment, as seen by a user. 


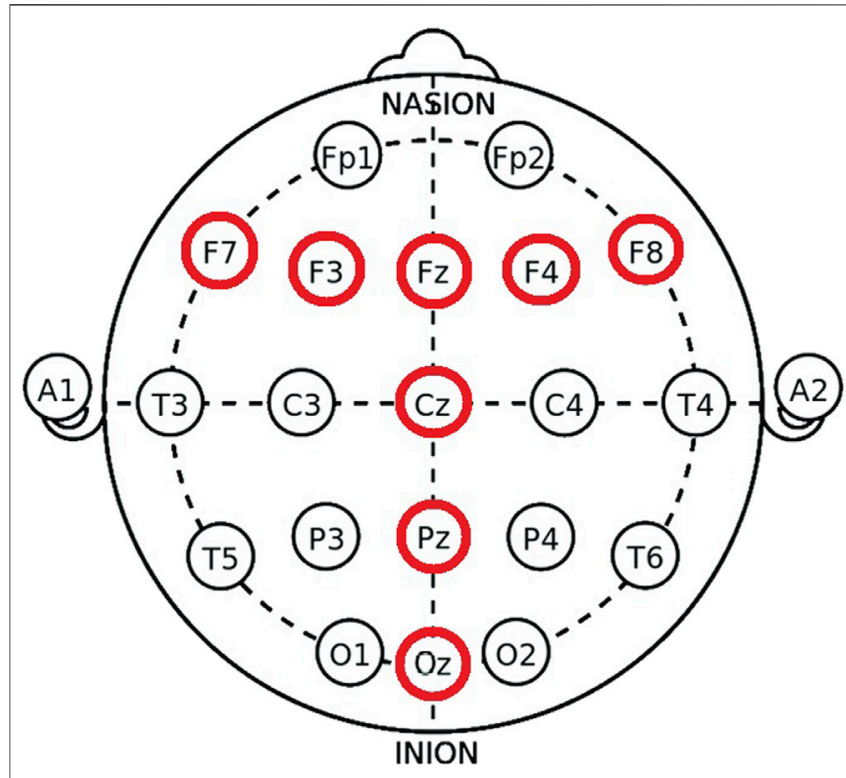

FIGURE 3 | 10-20 Diagram with red circles indicating the F7, F3, Fz, F4, $\mathrm{F} 8, \mathrm{Cz}, \mathrm{Pz}$ and $\mathrm{Oz}$ sites recorded during the study.

- Big spider near (BSN)-A big spider crawling near the participant's hand.

- Three spiders (3S)-Two different size spiders crawling in the box, with another descending down a web string.

- Swarm spiders (SS)-Twenty very small spiders crawling quickly around the box.

An additional two scenarios were created in which spiders touched the participant's hand:

- Web spider drop (WSD)-A small spider slowly crawling down a web above the participant's hand before dropping and remaining statically positioned on the participants hand for approximately $5 \mathrm{~s}$.

- Swarm spiders drop (SSD)-A $5 \mathrm{~s}$ wait before a series of twenty very small spiders fall from a chute on the top of the box; landing and crawling over the participants hand.

The WSD and SSD scenarios could also be enabled with tactile sensations from the Ultrahaptics board, denoted as $\mathrm{WSD}_{\mathrm{h}}$ and $\mathrm{SSD}_{\mathrm{h}}$, respectively. This resulted in a combined total of eleven possible scenarios.

The wireless Enobio eight from Neuroelectrics was used to gather EEG signals with eight $\mathrm{AgCl}$ coated dry electrodes in the F7, F3, Fz, F4, F8, Cz, Pz, and Oz positions, as shown in Figure 3. Two additional reference electrodes were located at the mastoid process location to enable signal noise to be filtered. EEG signals were recorded using the NIC2 software application with relevant events marked via a TCP marker stream from Unity 3D. Galvanic Skin Response (GSR) recordings were carried out using the wireless Shimmer 3 GSR + unit from Shimmer. Two $20 \mathrm{~mm}$ diameter, wet electrodes were placed on the palm of the nondominant hand for GSR measurements.

\subsection{Procedure}

Before any participant participated in the study they completed and returned an FSQ via email. Upon arrival in the laboratory each participant was provided with a consent form and study information sheet in compliance with the faculty board of ethics' regulations. When the participant was happy to proceed the physiological devices were attached and verified by observation of the real-time signals. Following this the participant was seated on a chair in front of the box with the front curtain obscuring the inside. There a VR HMD was set up and the participant was asked to orally provide a rating between 1 and 10 for their current state anxiety. Once the participant provided a number the baseline period commenced; where they remained seated for a total of $2 \mathrm{~m}$ whilst viewing an empty white canvas in VR.

Following the baseline phase, the curtain covering the front of the box was lifted and the virtual environment was switched to one containing a virtual box in the same location as the physical box. Each participant was given a chance to adapt to their environment during a hand positioning phase, usually lasting less than $30 \mathrm{~s}$, where they positioned their dominant hand at the target shown within the box in VR using feedback provided by the Leap Motion tracking device.

The repeating segment in the study involved the participant having their hand in the box with virtual spiders for $30 \mathrm{~s}$, as shown in Figure 4. At the end of this experience the participant was prompted for a 1-10 rating of their anxiety. After they responded a $10 \mathrm{~s}$ cooldown period was initiated; during which the participant viewed a white canvas. This procedure was then repeated until a total of nine spider scenarios had taken place in a random order. Participants had interactions with haptics in either the $\mathrm{WSD}_{\mathrm{h}}$ or $\mathrm{SSD}_{\mathrm{h}}$ scenarios. Scenarios were counterbalanced to ensure that the scenarios which could be provided with or without haptics were done in equal proportion.

On completion of the tests the physiological measurement devices and VR HMD were removed from the participant. After the experiment the participants completed the Slater-Usoh-Steed (SUS) Usoh et al. (2000) questionnaire on immersion and presence, provided a score for two questions, "On a scale of 1-10,

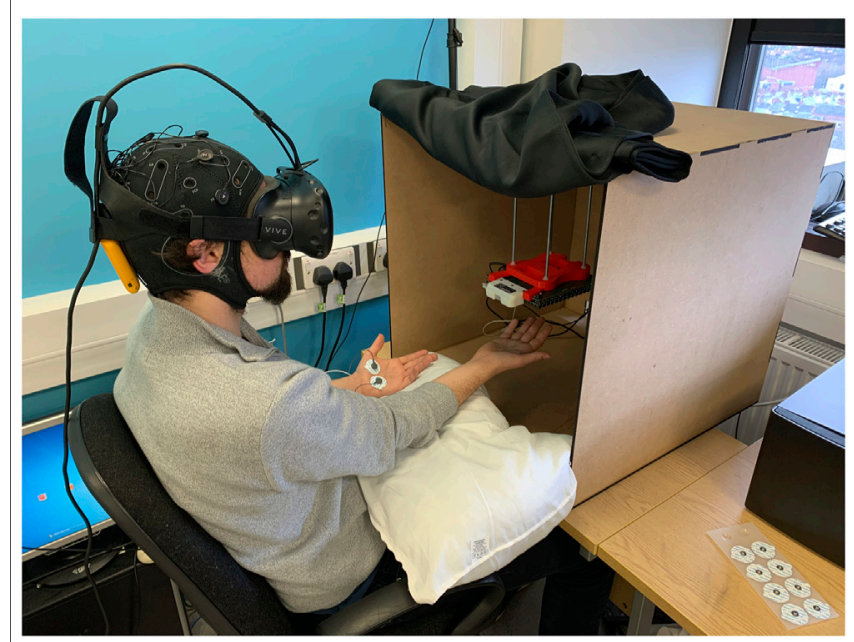

FIGURE 4 | Photo of apparatus setup with user in position. 
with one being very unrealistic and 10 being very realistic: How realistic would you say the haptics were for the spider?" and "On a scale of $1-10$, with one being very inaccurate and 10 being very accurate: How well do you think the position of the haptics matched that of the virtual spider?" and discussed their thoughts on the experience. In their discussion each participant was also asked to answer the question, "What could have increased your anxiety regarding the VR spiders?". Discussions were recorded and agreed upon by the participants.

\subsection{Measurements}

Similarly to many other studies Rinck and Becker (2007); Pflugshaupt et al. (2005); Teachman et al. (2001) participants' fear of spiders was assessed using the FSQ. This is an 18 item self-report measure where participants score their agreement on a seven-point Likert scale (zero = strongly disagree, six = strongly agree) for statements about spiders such as, "I would feel very nervous if I saw a spider now". The FSQ has been proven in the past to be effective in quantifying a person's fear of spiders Muris and Merckelbach (1996). In these studies a mean score of 89.1 in the FSQ is produced from phobic participants pre-treatment. A group of participants with at least a mid-level fear of spiders can be created using FSQ scores above 15 Cochrane et al. (2008).

Subjective self-reports of anxiety between 1 and 10 were provided by participants as the shortened version of the SUDS Wolpe (1990), a methodology employed in many studies Mystkowski et al. (2002); Benjamin et al. (2010); Heinssen et al. (1987); Nesse et al. (1985). Ratings were provided orally once before the baseline phase and following each spider scenario after it. This type of technique offers the cognitive evaluation that the participant makes of the stimulus situation or the cognitive evaluation of the emotional activation itself. To quantify levels of anxiety beyond those subjectively reported, two forms of objective measurements were introduced. GSR was used in the study as one of these objective indicators of anxiety, having already demonstrated its robustness and accuracy in the literature. Signals were recorded across the palm of the hand outside of the box. The data was segmented into windows for the baseline and spider scenarios. Mean values, reported in microsiemens $(\mu S)$, were derived for these windows; a method shown to be effective for other studies measuring anxiety in VR Meehan et al. (2002); Stinson and Bowman (2014); Meehan et al. (2002). Larger values indicate higher levels of stress and anxiety.

The other objective measurement technique used for anxiety in the study was dry-electrode EEG. This was selected due to its ability to measure across short periods of time, it not causing practical issues when used alongside GSR and evidence of it accurately measuring anxiety in the literature Wiedemann et al. (1999). EEG signals were collected at $500 \mathrm{~Hz}$ from the F3, F4, F7 and F8 sites using the 10-20 system Homan et al. (1987) after impedance checks showed resistance to be less than $20 \mathrm{kOhm}$, a threshold successfully demonstrated in previous studies Luck (2014); Gibson et al. (2018). Data was pre-processed in EEG Lab Delorme and Makeig (2004) with visual inspections of $2 \mathrm{~s}$, overlapped epochs performed for artefact rejection, a range recommended in the works of Smith et al. Smith et al. (2017). Epochs containing blink artefacts, flat lining, simple voltage threshold, and step functions were all removed during the epoch by epoch visual inspection. Power bands were estimated for each electrode in the alpha frequency $(8-13 \mathrm{~Hz})$ Davidson et al. (1990). Frontal asymmetry values were calculated by comparing log transformations of the left and right sides. This process was also carried out for the baseline and the different scenarios with spiders. Alpha power is inversely related to cortical activity Gollan et al. (2014) and asymmetry values can be used to show greater left side cortical activity; indicating emotions such as anxiety Wacker et al. (2003); Brouwer et al. (2011); Dennis and Solomon (2010). In our results lower asymmetry values indicate feelings of anxiety and withdrawal; whilst higher asymmetry values indicate emotions such as joy and exploration.

The level of presence has been shown to be effectively measured using the six question SUS questionnaire Usoh et al. (2000); Slater and Wilbur (1997); Slater et al. (1994). This is a questionnaire where participants are presented statements such as, "To what extent were there times during the experience when the virtual environment was the reality for you?" and provide an answer corresponding to a value on a Likert scale (0-6). The sum of the scores of the individual questions can be used to indicate a participant's sense of presence in VR. Participants completed the questionnaire immediately upon leaving the virtual environment.

\subsection{Analysis}

Statistical analysis was carried out using SPSS (v21). For withinsubjects factors, such as increase of anxiety from baseline, paired sample t-tests were used for comparisons. Wilcoxon signed-rank tests were used for data that failed the Shapiro-Wilk test for normality. Spearman rank correlations were calculated for correlations against non-parametric data sets such as FSQ scores. All tests were performed at the 95\% confidence level with $p<0.05$ being satisfied. Box and whisker plots are used to illustrate comparisons of anxiety level metrics between baseline periods and the spider scenarios. Within the plots; the T-bars indicate 95\% confidence interval extents, the edges of the box represent the upper and lower quartiles and the line within the box represents the median. Outliers are indicated with asterisks and circles.

\section{RESULTS}

\subsection{Change in Anxiety Levels}

A cut-off of 15 was used with the FSQ questionnaire to classify a group of 17 participants with atleast a mid-level fear of spiders, similarly to another study Wagener and Zettle (2011) that included a demonstration of spider exposure therapy. For subjective measures of anxiety comparisons of ratings were made between the pre-baseline rating and the mean average rating taken during the scenarios. For objective measures, comparisons were made between baseline readings and mean averages across scenarios. Results are illustrated in Figure 5.

The mean subjective anxiety rating pre-baseline was $2.12(\mathrm{SD}=$ 1.26). The participant mean subjective rating across all scenarios was 3.90 ( $\mathrm{SD}=1.77$ ). The distributions of ratings were found to be nonnormally distributed for the baseline and the aggregation across scenarios, with Shapiro-Wilk testing being W (35) $=0.783, p<0.001$ and $\mathrm{W}(35)=0.853, p<0.001$, respectively. A Wilcoxon signed-rank test was used to compare the groups due to the non-normal 

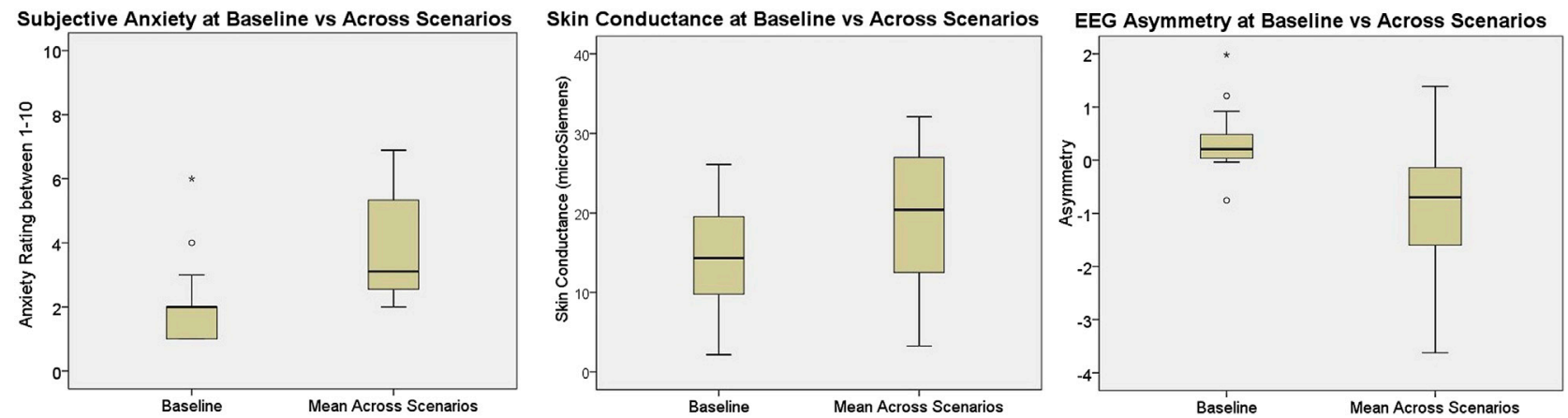

FIGURE 5| Box and whisker plots showing comparisons of baseline vs across scenarios for subjective, GSR and EEG anxiety metrics for participants with an FSQ score $>15$.

distribution of ratings. This showed there to be a statistically significant increase in anxiety $(Z=-3.52, p<0.001, d=0.85)$. Analysis of the GSR data provided mean values, measured in microSiemens, for skin conductance during the baseline stage (M $=14.96, \mathrm{SD}=6.62)$ and as mean average across all scenarios $(\mathrm{M}=$ $19.16, \mathrm{SD}=9.68)$. A paired sample $t$-test showed this difference to be statistically significant $(\mathrm{t}(16)=-2.87, p<0.001, d=1.11)$, indicating an increase in anxiety. EEG data was processed to provide asymmetry values indicating the difference in frontal-brain activity between left and right sides. This was carried out for the baseline stage $(M=0.35$, $\mathrm{SD}=0.61$ ) with a Shapiro-Wilk test showing the dataset to be nonnormally distributed, $\mathrm{W}(35)=0.849, p<0.001$. Similarly this was measured as mean average across scenarios $(\mathrm{M}=-0.96, \mathrm{SD}=1.31)$, with a Shaprio-Wilk test indicating the data is non-normally distributed, $\mathrm{W}(35)=0.917, p=0.02$. A Wilcoxon signed-rank test, used due to non-normal distribution, showed this decrease in asymmetry to be statistically significant $(\mathrm{Z}=-2.64, p=0.006, d=$ 0.64). Spearman's rank correlations between changes in physiological measurements and changes in subjective self-reports for anxiety were calculated. There were no correlations found between the overall change in physiological indicators and the change in self-reports from baseline to an aggregate of the scenarios. Spearmans rank correlations for GSR with subjective and EEG with subjective were; $r_{\mathrm{s}}=0 .-0.168$, $p=0.335$ and $r_{s}=0.086, p=0.647$, respectively.

\subsection{Relationship Between FSQ and Anxiety Induced}

Differences in subjective anxiety measures were calculated between baseline stages and averages across all scenarios in a particular participant's trial. This was done for all 35 participants. These differences were then correlated with participant FSQ scores to demonstrate that the anxiety was spider related and that the effectiveness of the system is correlated with the participant's fear of spiders. Evidence of this can be seen from Figure 6 which shows participants with higher FSQ scores generally reporting greater levels of anxiety than those with lower FSQ scores. Figure $\mathbf{6}$ also shows that only those with low FSQ scores experienced a decrease in anxiety levels from the baseline period. Correlations were calculated using Spearman's rank-order correlations due to non-normal distributions of the

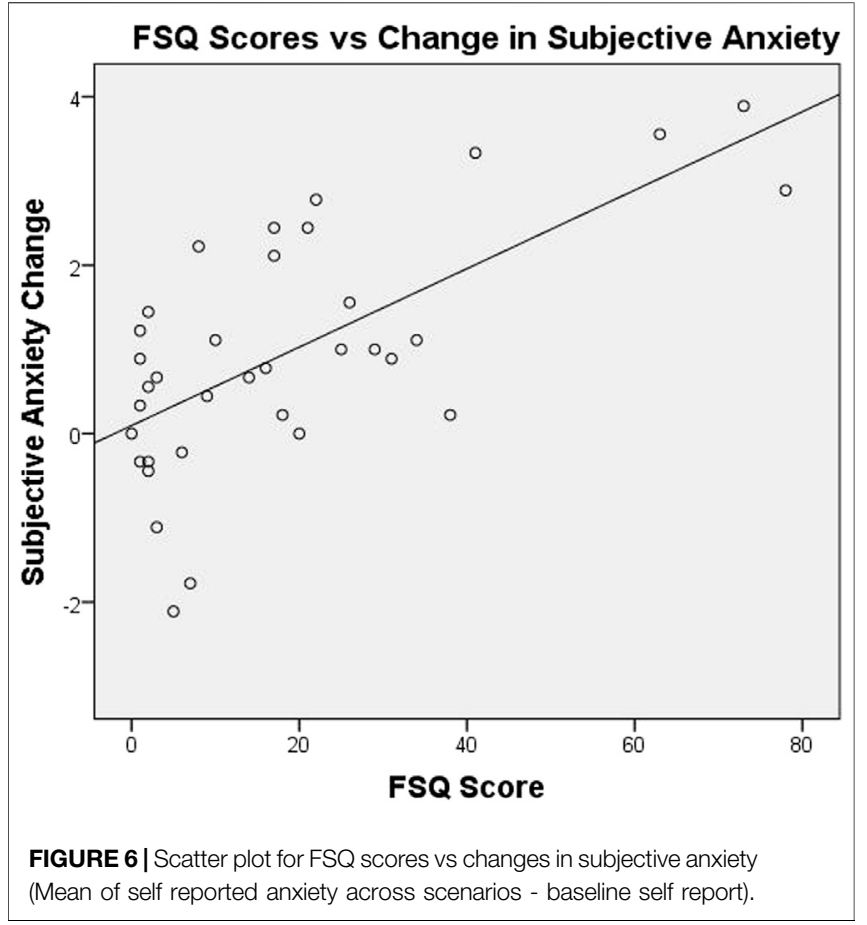

FSQ scores, Shapiro-Wilk test shows W $(35)=0.809, p<0.001$. A Spearman's correlation showed there to be a statistically significant, moderate and positive correlation between FSQ scores and change in subjective anxiety $\left(\mathrm{r}_{\mathrm{s}}=0.584, p<0.001\right)$.

\subsection{Relationship Between Presence and Anxiety Induced}

SUS questionnaires were used to provide a score between 0 and 36 for each of the 35 participants' sense of presence $($ Median $=22$, Quartiles $=17,25)$. Scores for participants were correlated with their change in anxiety, measured subjectively as the difference between self-reports of anxiety from baseline to the average of their self-reported anxiety across the trial's scenarios. These scores are shown plotted against the anxiety changes in 


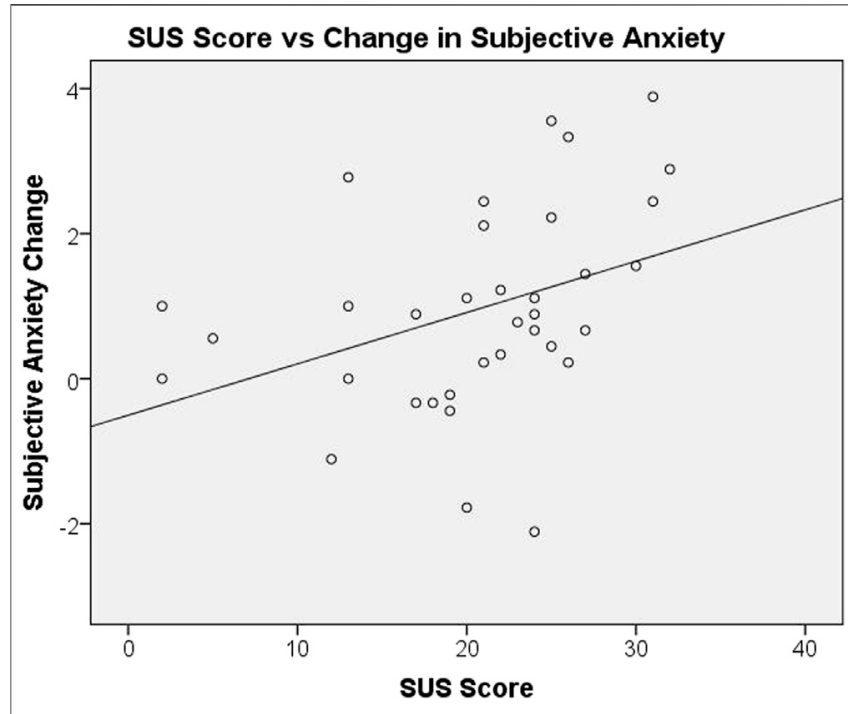

FIGURE 7 | Scatter plot for SUS scores vs changes in subjective anxiety (Mean of self reported anxiety across scenarios - baseline self report).

Figure 7. Spearman's rank-order correlations were used due to the non-parametric SUS scores. A Spearman's correlation showed there to be a moderate, positive and statistically significant correlation between level of presence and increase in selfreported anxiety $\left(r_{s}=0.490, p=0.003\right)$.

\subsection{The Effect of Haptics on Anxiety}

The effect of haptics on participants' self-reported anxiety levels was measured for spider scenarios which alternated between having haptics enabled and not enabled across all participants, $\mathrm{WSD}, \mathrm{WSD}_{\mathrm{h}}, \mathrm{SSD}$ and $\mathrm{SSD}_{\mathrm{h}}$. These anxiety scores can be seen in Figure 8. Shapiro-Wilk tests showed anxiety scores to be nonnormally distrbuted for WSD W $(18)=0.790, p=0.001$, SSD W $(17)=0.882, p=0.034$ and $\mathrm{SSD}_{\mathrm{h}} \mathrm{W}(18)=0.874, p=0.021$. The $\mathrm{WSD}_{\mathrm{h}}$ sample was found to be normally distributed in the ShapiroWilk test, $\mathrm{W}(17)=0.921, p=0.152$. However, Mann-Whitney U
Tests are used to compare between the independent samples due to the non-normal distributions of the other scenarios.

Median anxiety scores for WSD and $\mathrm{WSD}_{\mathrm{h}}$ were three and four; the difference between the groups was found to be non-statistically significant, $\mathrm{U}=133, p=0.525$. Median anxiety scores for SSD and $\mathrm{SSD}_{\mathrm{h}}$ were three and 3.5; the difference between the groups was also found to be non-statistically significant, $U=108, p=0.143$.

\subsection{Quantitative Feedback on Haptics Accuracy and Realism}

In response to the question, "On a scale of 1-10, with one being very unrealistic and 10 being very realistic: How realistic would you say the haptics were for the spider?", scores were collected across all 35 participants (Median $=6$, Quartiles $=5,7$ ). Scores were also collected in response to the second question, "On a scale of $1-10$, with one being very innacurate and 10 being very accurate: How well do you think the position of the haptics matched that of the virtual spider?" (Median $=8$, Quartiles $=7,9$ ). These scores for realism and accuracy can be seen in Figure 9.

\section{DISCUSSION}

One of the essential capabilities of VRET is being able to provide a virtual environment where people can confront anxiety induced by representations of their phobias in as realistic a way as possible. If the VR application used within the study was unable to produce anxiety for those who were afraid of spiders it would question the suitability of similar systems for VRET as they would not provide users with a medium through which to practice anxiety reducing techniques.

Most of the existing studies involving spiders in VR focus on observing how successful arachnophobia VRET is in treating those with phobias. However, the participants in this study did not have a clinically significant phobia and the intention was not to treat any fear they had of spiders within the session. Instead the objective was to measure how much of an effect the VR spiders could have on anxiety levels in general. If the system could
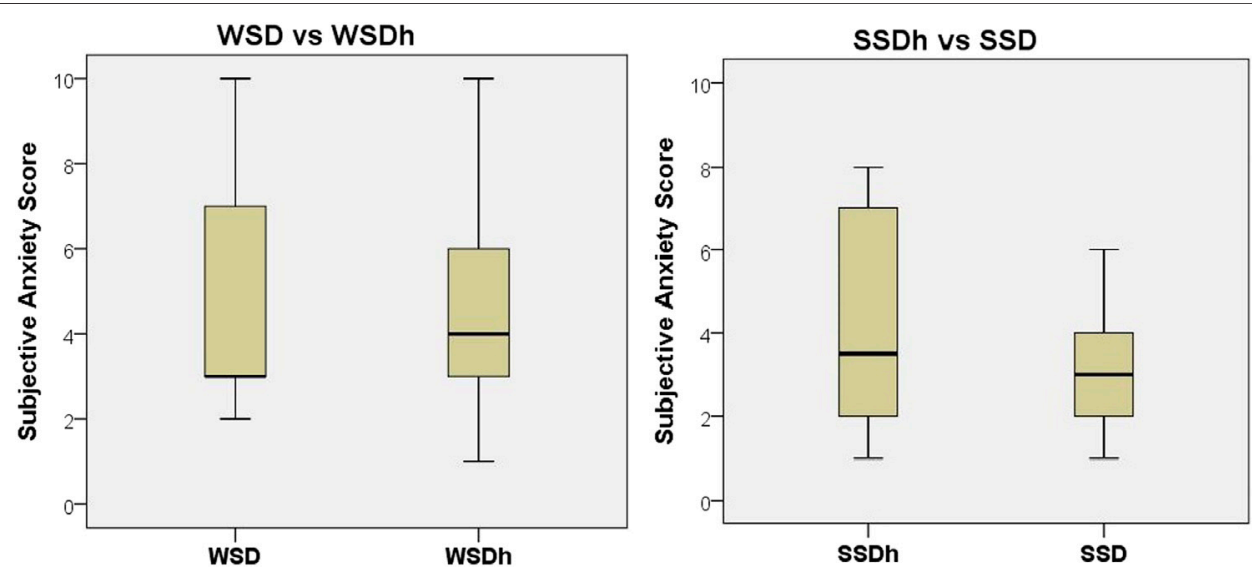

FIGURE 8 | Box and whisker plots showing comparisons of self-reported anxiety scores for two scenarios with and without haptics. 

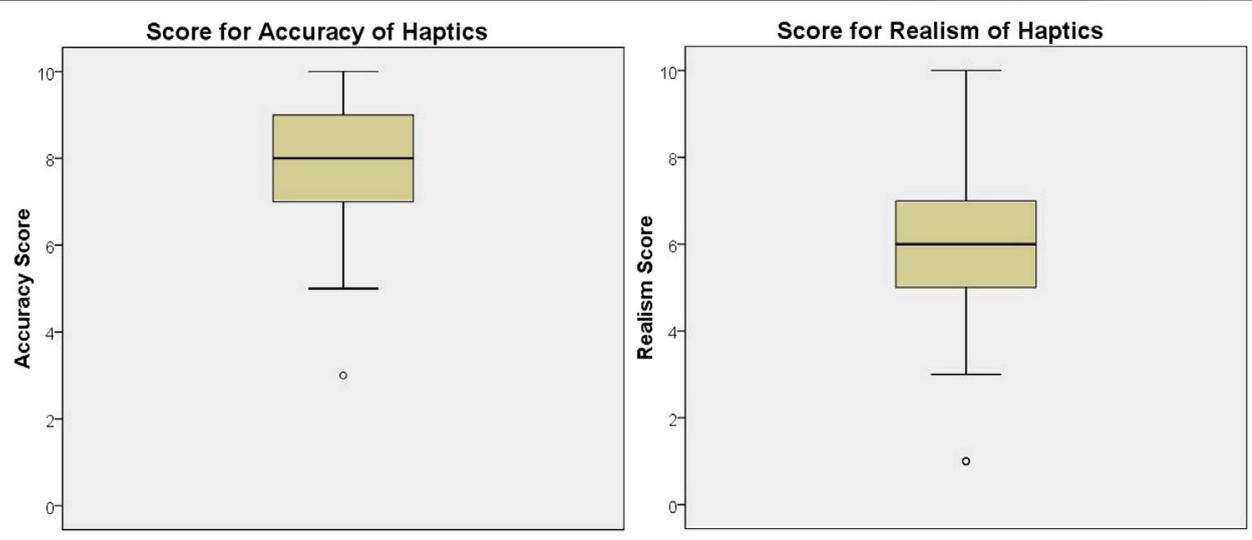

FIGURE 9 | Box and whisker plots showing scores for accuracy and realism of haptics.

effectively enhance the sense of anxiety within the participant sample the system could have an extensive audience of people with ranging levels of fear of spiders who could benefit. From the results it is clear that the system was indeed capable of inducing anxiety within the 17 participants with an FSQ score greater than 15 . Both the objective measurements, as well as the subjective selfreports, of anxiety showed a statistically significant increase from baseline to the mean anxiety of the VR spider scenarios.

There can be many factors which contribute to the anxiety measures for participants. However, the moderate correlation, which was shown to be statistically significant, between FSQ scores and the anxiety induced within the experience suggests that this anxiety level is being modulated by participants' fear of spiders. Therefore, the proof of concept system can be expected to affect those who are more afraid of spiders than those who are not and the spider related stimuli is playing a large role in this. The implication of this is that our application has high specificity in inducing anxiety in response to the presence of virtual spiders with enhanced realism (the inclusion of ultrasound haptics) and it is not simply increasing anxiety through confounding factors, such as wearing a VR headset or additional physiological measurement equipment.

Self-reported anxiety scores for the WSD, $\mathrm{WSD}_{\mathrm{h}}$, SSD and $\mathrm{SSD}_{\mathrm{h}}$ showed there to be slightly higher median scores when haptics were included. However, these differences were found to be non-statistically significant. This is unsurprising as the comparison of haptics vs non-haptics on a scenario is made between subjects with varying levels of fear of spiders. The lack of statistically significant difference between the haptic and nonhaptic groups suggests that the inclusion of the haptics did not have a major effect on participant anxiety. Although initial measures into the sole effect of the presence of haptics on anxiety are reported in this study, future within-subject studies comparing haptics vs non-haptic scenarios are encouraged to produce more conclusive findings on the matter.

Quantitative feedback was gathered from participants on their impressions of their ultrasonic haptic feedback. Scores on the question regarding the accuracy of the haptics indicated that participants found the ultrasonic haptics to be accurate in corresponding to the location of the virtual spiders. This suggests that the physical apparatus set-up presented in the study may be valid to use in conjuncture with virtual environments to accurately contextualise haptic sensations with visual counterparts and that higher precision tracking technologies are not necessary to achieve good results. Results from the participants' quantitative feedback found the realism of the ultrasonic haptics to be fairly neutral overall. Participants were mixed in their opinions on how realistic the haptics felt, resulting in realism scores as low as one and as high as 10 . This indicates that whilst the haptic methodology of ultrasonic feedback in circular sensations may be accurate in its location, it does not guarantee highly realistic sensations. Initial feedback on the realism of ultrahaptics sensations for spiders has been disseminated. However, user studies comparing ultrasonic feedback platforms and alternative technologies are recommended to produce benchmarks that can aid developers in selecting haptics technologies for various applications.

During the discussions following the VR experience many of the participants remarked that they were surprised at just how effective the VR spiders were in creating anxiety within them. When asked, "what could have increased your anxiety regarding the VR spiders?", there were common themes in the responses from the 35 participants. 15 of the participants said they would have been made more anxious by the presence of larger spiders, making this the most common suggestion. The larger spiders used in the study were of approximately $80 \mathrm{~mm}$ diameter, intended to replicate a large house spider. However, from this response it appears that there is scope for a more anxiety enhancing application using larger species of spider, such as tarantulas. There were also suggestions surrounding the behaviour of the spiders, with nine participants indicating faster moving spiders would have increased anxiety and six participants suggesting jumping spiders.

Considering VRET applications are intended to recreate aspects of phobias the real world, a goal of the design of such applications should be the inclusion of highly immersive methodologies. The results of the SUS questionnaire on immersion and presence in the study indicate the experience was fairly immersive, with a median score of 22 out of 36. Post-VR discussions with participants on their experience highlighted that some of the participants did not think the virtual room they were in was relatable enough, with 
cobbled walls and floors. There was no overall consensus regarding the graphical qualities of the experience; many participants stated they would have preferred higher resolution models, whilst many others were surprised at how realistic they looked.

The statistically significant, moderate correlation between SUS scores for overall change in anxiety $\left(r_{s}=0.490, p=0.003\right)$ indicates that there is certainly a relationship between how immersive participants found the system to be and how much of an effect it had on their anxiety. These results are in line with many other studies where significant correlations have been found between inducing anxiety and immersion Alsina-Jurnet et al. (2011); Pallavicini et al. (2013); Kwon et al. (2013); Robillard et al. (2003); Bouchard et al. (2008); Diemer et al. (2015); Krijn et al. (2004). This is further evidence of how significant a role immersion has in VRET applications, as well as other VR experiences with the intention to induce anxiety (e.g. VR Training, VR Gaming, etc ... ).

\section{LIMITATIONS AND FURTHER WORK}

One of the limitations of the study is the use of participants who were non-phobic to determine the effectiveness of a proof of concept VRET demonstration. Typical users of such a system would usually have some form of phobia, making it likely there would be a greater effect on their anxiety. A more ideal demographic of participants for the study would be those with arachnophobia, the intended end-user of such a system. However, access to large numbers of suitable participants with the phobia can be challenging and usage of non-phobic volunteers still produces meaningful results and implications.

Another limitation is the small sample size for data corresponding to the haptic spider scenarios, with one haptics condition being run per participant trial. Although the overall system was effective in modulating anxiety, there were no differences found between scenarios' haptic and non-haptic counterparts. Therefore, these findings do not suggest that the ultrasonic feedback had a major role to play in affecting anxiety levels. This study has introduced the use of ultrasonic haptics for spider sensations with initial findings, but further studies are required to gather additional insight into their exclusive effectiveness and potential therapeutic value.

All of the measures for state-anxiety indicated overall increase across the study. However, there was still an absence of correlation between the physiological measurements and self-reports. One possible reason for this is experimenter bias, whereby social desirability may play a factor in participants self-reports and questionnaires. This is a common problem when using subjective measures and further indicates the necessity for objective measures to be taken alongside subjective ones.

\section{CONCLUSION}

A proof of concept demonstration of a multisensory, VRET environment with virtual spiders has been presented for an immersive user experience. This system has enabled a user study to be conducted demonstrating the effectiveness of VR to enhance the sense of anxiety within users who reported at least a mid-level fear of spiders. Within the study the system was found to be clearly effective, with skin conductance, EEG and subjective results all showing statistically significant increases in anxiety for the aforementioned participants during their experiences. A correlation between FSQ scores and change in anxiety indicates the anxiety created was related to the presence of the virtual spiders and not other confounding factors. Our findings suggest that overall system was highly effective in enhancing the sense of anxiety for those who are somewhat afraid of spiders. However, preliminary measures on the effect of the presence of haptics on anxiety levels indicate they did not enhance anxiety. Users provided quantitative feedback on the accuracy and realism of the ultrasonic haptics and whilst the majority found the system to be accurate in locating the haptics, opinions on the realism of them were mixed with a median score of $6 / 10$. Additionally, a correlation between change in anxiety and sense of presence adds further evidence of the significance of immersion in VRET design.

\section{DATA AVAILABILITY STATEMENT}

The raw data supporting the conclusions of this article will be made available by the authors, without undue reservation.

\section{ETHICS STATEMENT}

The studies involving human participants were reviewed and approved by the Faculty of Engineering and Physical Sciences Research Ethics Committee. Queen's University (Belfast). The patients/participants provided their written informed consent to participate in this study. Written informed consent was obtained from the individual(s) for the publication of any potentially identifiable images or data included in this article.

\section{AUTHOR CONTRIBUTIONS}

DB developed the software/hardware setup, implemented the study and analysed the results. ZG contributed to the design of the study and analysis of the EEG data. FM contributed by developing prototype software. KR provided guidance on various aspects, such as study design. All authors contributed to the article and approved the submitted version.

\section{FUNDING}

This work was partially supported by the Centre for Intelligent Autonomous Manufacturing Systems (i-AMS).

\section{ACKNOWLEDGMENTS}

We would like to thank Dr Paul Best in his guidance on clinical therapy practices surrounding arachnophobia. We would also like to thank the participants who participated in the study for their time and bravery. 


\section{REFERENCES}

Alsina-Jurnet, I., Gutiérrez-Maldonado, J., and Rangel-Gómez, M.-V. (2011). The Role of Presence in the Level of Anxiety Experienced in Clinical Virtual Environments. Comput. Hum. Behav. 27, 504-512. doi:10.1016/ j.chb.2010.09.018

Benjamin, C. L., O'Neil, K. A., Crawley, S. A., Beidas, R. S., Coles, M., and Kendall, P. C. (2010). Patterns and Predictors of Subjective Units of Distress in Anxious Youth. Behav. Cogn. Psychother. 38, 497-504. doi:10.1017/s1352465810000287

Bouchard, S., St-Jacques, J., Robillard, G., and Renaud, P. (2008). Anxiety Increases the Feeling of Presence in Virtual Reality. Presence: Teleoperators and Virtual Environments 17, 376-391. doi:10.1162/pres.17.4.376

Boyd, J. H., Rae, D. S., Thompson, J. W., Burns, B. J., Bourdon, K., Locke, B. Z., et al. (1990). Phobia: Prevalence and Risk Factors. Soc. Psychiatry Psychiatr. Epidemiol. 25, 314-323. doi:10.1007/BF00782887

Brouwer, A.-M., Neerincx, M., Kallen, V., van der Leer, L., and ten Brinke, M. (2011). Eeg Alpha Asymmetry, Heart Rate Variability and Cortisol in Response to Virtual Reality Induced Stress. J. CyberTherapy Rehabil. 4, 83-99.

Carlin, A. S., Hoffman, H. G., and Weghorst, S. (1997). Virtual Reality and Tactile Augmentation in the Treatment of Spider Phobia: a Case Report. Behav. Res. Ther. 35, 153-158. doi:10.1016/S0005-7967(96)00085-X

Carter, T., Seah, S., Long, B., Drinkwater, B., and Subramanian, S. (2013). Ultrahaptics: Multi-point Mid-air Haptic Feedback for Touch Surfaces, 505-514. doi:10.1145/2501988.2502018

Cavrag, M., Larivière, G., Cretu, A.-M., and Bouchard, S. (2014). "Interaction with Virtual Spiders for Eliciting Disgust in the Treatment of Phobias," in 2014 IEEE International Symposium on Haptic, Audio and Visual Environments and Games (HAVE) Proceedings (IEEE), 29-34. doi:10.1109/HAVE.2014.6954327

Cochrane, A., Barnes-Holmes, D., and Barnes-Holmes, Y. (2008). The PerceivedThreat Behavioral Approach Test (Pt-bat): Measuring Avoidance in High-, Mid-, and Low-Spider-Fearful Participants. Psychol. Rec. 58, 585-596. doi:10.1007/BF03395639

Davidson, R. J., Ekman, P., Saron, C. D., Senulis, J. A., and Friesen, W. V. (1990). Approach-withdrawal and Cerebral Asymmetry: Emotional Expression and Brain Physiology: I. J. Personal. Soc. Psychol. 58, 330-341. doi:10.1037/00223514.58.2.330

Delorme, A., and Makeig, S. (2004). Eeglab: an Open Source Toolbox for Analysis of Single-Trial Eeg Dynamics Including Independent Component Analysis. J. Neurosci. Methods 134, 9-21. doi:10.1016/j.jneumeth.2003.10.009

Dennis, T. A., and Solomon, B. (2010). Frontal Eeg and Emotion Regulation: Electrocortical Activity in Response to Emotional Film Clips Is Associated with Reduced Mood Induction and Attention Interference Effects. Biol. Psychol. 85, 456-464. doi:10.1016/j.biopsycho.2010.09.008

Depla, M. F. I. A., ten Have, M. L., van Balkom, A. J. L. M., and de Graaf, R. (2008). Specific Fears and Phobias in the General Population: Results from the netherlands Mental Health Survey and Incidence Study (Nemesis). Soc. Psychiat Epidemiol. 43, 200-208. doi:10.1007/s00127-007-0291-z

Diemer, J., Alpers, G. W., Peperkorn, H. M., Shiban, Y., and Mã1/4hlberger, A. (2015). The Impact of Perception and Presence on Emotional Reactions: a Review of Research in Virtual Reality. Front. Psychol. 6, 26. doi:10.3389/ fpsyg.2015.00026

Garcia-Palacios, A., Botella, C., Hoffman, H., and Fabregat, S. (2007). Comparing Acceptance and Refusal Rates of Virtual Reality Exposure vs. In Vivo Exposure by Patients with Specific Phobias. CyberPsychology Behav. 10, 722-724. doi:10.1089/cpb.2007.9962

Garcia-Palacios, A., Hoffman, H., Carlin, A., Furness, T. A., and Botella, C. (2002). Virtual Reality in the Treatment of Spider Phobia: a Controlled Study. Behav. Res. Ther. 40, 983-993. doi:10.1016/s0005-7967(01)00068-7

Gibson, Z., Joseph, B., Roger, M., Murphy, B., and Marzano, A. (2018). "Use of Dry Electrode Electroencephalography (Eeg) to Monitor Pilot Workload and Distraction Based on P300 Responses to an Auditory Oddball Task," in International Conference on Applied Human Factors and Ergonomics (Springer), 14-26. doi:10.1007/978-3-319-94866-9_2

Gollan, J. K., Hoxha, D., Chihade, D., Pflieger, M. E., Rosebrock, L., and Cacioppo, J. (2014). Frontal Alpha Eeg Asymmetry before and after Behavioral Activation Treatment for Depression. Biol. Psychol. 99, 198-208. doi:10.1016/ j.biopsycho.2014.03.003
Heinssen, R. K., Glass, C. R., and Knight, L. A. (1987). Assessing Computer Anxiety: Development and Validation of the Computer Anxiety Rating Scale. Comput. Hum. Behav. 3, 49-59. doi:10.1016/0747-5632(87)90010-0

Hoffman, H. G., Garcia-Palacios, A., Carlin, A., Furness III, T. A., and BotellaArbona, C. (2003). Interfaces that Heal: Coupling Real and Virtual Objects to Treat Spider Phobia. Int. J. Human-Computer Interaction 16, 283-300. doi:10.1207/s15327590ijhc1602_08

Hoffman, H. G., Groen, J., Rousseau, A., Hollander, A. L., Winn, W., Wells, M. J. et al. (1996). Tactile Augmentation: Enhancing Presence in Virtual Reality with Tactile Feedback from Real Objects.

Hoffman, H. G., Hollander, A., Schroder, K., Rousseau, S., and Furness, T. (1998). Physically Touching and Tasting Virtual Objects Enhances the Realism of Virtual Experiences. Virtual Reality 3, 226-234. doi:10.1007/BF01408703

Hoffman, H. G. (1998). "Physically Touching Virtual Objects Using Tactile Augmentation Enhances the Realism of Virtual Environments," in Proceedings of the Virtual Reality Annual International Symposium (USA: IEEE Computer Society), 59.

Homan, R. W., Herman, J., and Purdy, P. (1987). Cerebral Location of International 10-20 System Electrode Placement. Electroencephalography Clin. Neurophysiol. 66, 376-382. doi:10.1016/0013-4694(87)90206-9

Hoshi, T., Takahashi, M., Iwamoto, T., and Shinoda, H. (2010). Noncontact Tactile Display Based on Radiation Pressure of Airborne Ultrasound. IEEE Trans. Haptics 3, 155-165. doi:10.1109/TOH.2010.4

Iwamoto, T., Tatezono, M., and Shinoda, H. (2008). "Non-contact Method for Producing Tactile Sensation Using Airborne Ultrasound," in Haptics: Perception, Devices and Scenarios. Editor M. Ferre (Berlin, Heidelberg: Springer Berlin Heidelberg), 504-513.

Iza, M., Olfson, M., Vermes, D., Hoffer, M., Wang, S., and Blanco, C. (2013). Probability and Predictors of First Treatment Contact for Anxiety Disorders in the United States. J. Clin. Psychiatry 74, 1093-1100. doi:10.4088/ JCP. $13 \mathrm{~m} 08361$

Juan, M. C., and Pérez, D. (2010). Using Augmented and Virtual Reality for the Development of Acrophobic Scenarios. Comparison of the Levels of Presence and Anxiety. Comput. Graphics 34, 756-766. doi:10.1016/j.cag.2010.08.001

Krijn, M., Emmelkamp, P. M. G., Biemond, R., de Wilde de Ligny, C., Schuemie, M. J., and van der Mast, C. A. P. G. (2004). Treatment of Acrophobia in Virtual Reality: The Role of Immersion and Presence. Behav. Res. Ther. 42, 229-239. doi:10.1016/S0005-7967(03)00139-6

Kurscheidt, M., Ostapchuck, V., Sottek, F., Rauh, S. F., and Meixner, G. (2019). "Augmented Reality Exposure Therapy with Tactile Feedback for Small Animal Phobia : Hardware Concept and User Study Design," in 2019 IEEE International Conference on Healthcare Informatics (ICHI) (IEEE), 1-7. doi:10.1109/ICHI.2019.8904800

Kwon, J. H., Powell, J., and Chalmers, A. (2013). How Level of Realism Influences Anxiety in Virtual Reality Environments for a Job Interview. Int. J. HumanComputer Stud. 71, 978-987. doi:10.1016/j.ijhcs.2013.07.003

Lombard, M. (2000). Resources for the Study of Presence: Presence Explication.

Long, B., Seah, S. A., Carter, T., and Subramanian, S. (2014). Rendering Volumetric Haptic Shapes in Mid-air Using Ultrasound. ACM Trans. Graph. 33. doi:10.1145/2661229.2661257

Luck, S. (2014). An Introduction to the Event-Related Potential Technique. MIT Press.

Meehan, M., Insko, B., Whitton, M., and Brooks, F. P., Jr (2002). Physiological Measures of Presence in Stressful Virtual Environments. ACM Trans. Graphics 21, 1. doi: $10.1145 / 566570.566630$

Muris, P., and Merckelbach, H. (1996). A Comparison of Two Spider Fear Questionnaires. J. Behav. Ther. Exp. Psychiatry 27, 241-244. doi:10.1016/ S0005-7916(96)00022-5

Mystkowski, J. L., Craske, M. G., and Echiverri, A. M. (2002). Treatment Context and Return of Fear in Spider Phobia. Behav. Ther. 33, 399-416. doi:10.1016/ S0005-7894(02)80035-1

Nesse, R. M., Curtis, G. C., Thyer, B. A., McCann, D. S., Huber-Smith, M. J., and Knopf, R. F. (1985). Endocrine and Cardiovascular Responses during Phobic Anxiety. Psychosomatic Med. 47, 320-332. doi:10.1097/00006842-19850700000002

Ollendick, T. H., and Davis, T. E., III (2004). Empirically Supported Treatments for Children and Adolescents: Where to from Here? Clin. Psychol. Sci. Pract. 11, 289-294. doi:10.1093/clipsy.bph083 
Opriş, D., Pintea, S., García-Palacios, A., Botella, C., Szamosközi, Ş., and David, D. (2012). Virtual Reality Exposure Therapy in Anxiety Disorders: A Quantitative Meta-Analysis. Depress. Anxiety 29, 85-93. doi:10.1002/da.20910

Pallavicini, F., Cipresso, P., Raspelli, S., Grassi, A., Serino, S., Vigna, C., et al. (2013). Is Virtual Reality Always an Effective Stressors for Exposure Treatments? Some Insights from a Controlled Trial. BMC psychiatry 13, 52. doi:10.1186/1471244X-13-52

Peperkorn, H. M., and Mühlberger, A. (2013). The Impact of Different Perceptual Cues on Fear and Presence in Virtual Reality. Stud. Health Technol. Inform. 191, 75-79. doi:10.3233/978-1-61499-282-0-75

Pflugshaupt, T., Mosimann, U. P., Wartburg, R. v., Schmitt, W., Nyffeler, T., and Müri, R. M. (2005). Hypervigilance-avoidance Pattern in Spider Phobia. J. Anxiety Disord. 19, 105-116. doi:10.1016/j.janxdis.2003.12.002

Powers, M. B., and Emmelkamp, P. M. G. (2008). Virtual Reality Exposure Therapy for Anxiety Disorders: A Meta-Analysis. J. Anxiety Disord. 22, 561-569. doi:10.1016/j.janxdis.2007.04.006

Price, M., and Anderson, P. (2007). The Role of Presence in Virtual Reality Exposure Therapy. J. Anxiety Disord. 21, 742-751. doi:10.1016/ j.janxdis.2006.11.002

Rinck, M., and Becker, E. S. (2007). Approach and Avoidance in Fear of Spiders. J. Behav. Ther. Exp. Psychiatry 38, 105-120. doi:10.1016/j.jbtep.2006.10.001

Riva, G., Mantovani, F., Capideville, C. S., Preziosa, A., Morganti, F., Villani, D., et al. (2007). Affective Interactions Using Virtual Reality: The Link between Presence and Emotions. CyberPsychology Behav. 10, 45-56. doi:10.1089/ cpb.2006.9993

Robillard, G., Bouchard, S., Fournier, T., and Renaud, P. (2003). Anxiety and Presence during Vr Immersion: A Comparative Study of the Reactions of Phobic and Non-phobic Participants in Therapeutic Virtual Environments Derived from Computer Games. CyberPsychology Behav. 6, 467-476. doi:10.1089/109493103769710497

Rothbaum, B. O., Hodges, L. F., Kooper, R., Opdyke, D., Williford, J. S., and North, M. N. (1995). Effectiveness of Computer-Generated (Virtual Reality) Graded Exposure in the Treatment of Acrophobia. Ajp 152 (4), 626-628. doi:10.1176/ ajp.152.4.626

Segal, R., Bhatia, M., and Drapeau, M. (2011). Therapists' Perception of Benefits and Costs of Using Virtual Reality Treatments. Cyberpsychology, Behav. Soc. Networking 14, 29-34. doi:10.1089/cyber.2009.0398

Slater, M., Usoh, M., and Steed, A. (1994). Depth of Presence in Virtual Environments. Presence: Teleoperators \& Virtual Environments 3, 130-144. doi:10.1162/pres.1994.3.2.130

Slater, M., and Wilbur, S. (1997). A Framework for Immersive Virtual Environments (FIVE): Speculations on the Role of Presence in Virtual Environments. Presence: Teleoperators \& Virtual Environments 6, 603-616. doi:10.1162/pres.1997.6.6.603

Smith, E. E., Reznik, S. J., Stewart, J. L., and Allen, J. J. B. (2017). Assessing and Conceptualizing Frontal Eeg Asymmetry: An Updated Primer on Recording, Processing, Analyzing, and Interpreting Frontal Alpha Asymmetry. Int. J. Psychophysiology 111, 98-114. doi:10.1016/j.ijpsycho.2016.11.005

Stinson, C., and Bowman, D. A. (2014). Feasibility of Training Athletes for HighPressure Situations Using Virtual Reality. IEEE Trans. Vis. Comput. Graphics 20, 606-615. doi:10.1109/TVCG.2014.23
Stinson, F. S., Dawson, D. A., Patricia Chou, S., Smith, S., Goldstein, R. B., June Ruan, W., et al. (2007). The Epidemiology of Dsm-Iv Specific Phobia in the usa: Results from the National Epidemiologic Survey on Alcohol and Related Conditions. Psychol. Med. 37, 1047-1059. doi:10.1017/ S0033291707000086

Szymanski, J., and O'Donohue, W. (1995). Fear of Spiders Questionnaire. J. Behav. Ther. Exp. Psychiatry 26, 31-34. doi:10.1016/0005-7916(94)00072-T

Teachman, B. A., Gregg, A. P., and Woody, S. R. (2001). Implicit Associations for Fear-Relevant Stimuli Among Individuals with Snake and Spider Fears. J. Abnormal Psychol. 110, 226-235. doi:10.1037/0021-843x.110.2.226

Turner, W. A., and Casey, L. M. (2014). Outcomes Associated with Virtual Reality in Psychological Interventions: where Are We Now? Clin. Psychol. Rev. 34, 634-644. doi:10.1016/j.cpr.2014.10.003

Usoh, M., Catena, E., Arman, S., and Slater, M. (2000). Using Presence Questionnaires in Reality. Presence: Teleoperators and Virtual Environments 9. doi:10.1162/105474600566989

Vahia, V. (2013). Diagnostic and Statistical Manual of Mental Disorders 5: A Quick Glance. Indian J. Psychiatry 55, 220-223. doi:10.4103/0019-5545.117131

Wacker, J., Heldmann, M., and Stemmler, G. (2003). Separating Emotion and Motivational Direction in Fear and Anger: Effects on Frontal Asymmetry. Emotion 3, 167-193. doi:10.1037/1528-3542.3.2.167

Wagener, A. L., and Zettle, R. D. (2011). Targeting Fear of Spiders with Control-, Acceptance-, and Information-Based Approaches. Psychol. Rec. 61, 77-91. doi:10.1007/BF03395747

Wiedemann, G., Pauli, P., Dengler, W., Lutzenberger, W., Birbaumer, N., and Buchkremer, G. (1999). Frontal Brain Asymmetry as a Biological Substrate of Emotions in Patients with Panic Disorders. Arch. Gen. Psychiatry 56 (1), 78-84. doi:10.1001/archpsyc.56.1.78

Wolpe, J. (1990). "The Practice of Behavior Therapy," in Pergamon General Psychology Series; PGPS-1 (Pergamon Press).

Yeh, S.-C., Li, Y.-Y., Zhou, C., Chiu, P.-H., and Chen, J.-W. (2018). Effects of Virtual Reality and Augmented Reality on Induced Anxiety. IEEE Trans. Neural Syst. Rehabil. Eng. 26, 1345-1352. doi:10.1109/TNSRE.2018.2844083

Conflict of Interest: The authors declare that the research was conducted in the absence of any commercial or financial relationships that could be construed as a potential conflict of interest.

Publisher's Note: All claims expressed in this article are solely those of the authors and do not necessarily represent those of their affiliated organizations, or those of the publisher, the editors and the reviewers. Any product that may be evaluated in this article, or claim that may be made by its manufacturer, is not guaranteed or endorsed by the publisher.

Copyright (c) 2021 Brice, Gibson, McGuinness and Rafferty. This is an open-access article distributed under the terms of the Creative Commons Attribution License (CC $B Y)$. The use, distribution or reproduction in other forums is permitted, provided the original author(s) and the copyright owner(s) are credited and that the original publication in this journal is cited, in accordance with accepted academic practice. No use, distribution or reproduction is permitted which does not comply with these terms. 